\title{
Bandwidth Exchange as an Incentive for Relaying
}

\author{
Dan Zhang*, Ömer Ileri ${ }^{\dagger}$, Narayan Mandayam ${ }^{\ddagger}$ \\ $*$ WINLAB, Rutgers University \\ 671 Route 1 South, North Brunswick, NJ 08902 \\ Email: \{bacholic, narayan\}@winlab.rutgers.edu \\ †Wireless@KTH, Royal Institute of Technology, Electrum 418, SE-164 40 Kista, Sweden \\ Email: omer.ileri@radio.kth.se
}

\begin{abstract}
Relay cooperation has been recognized as an important mechanism to enhance connectivity and throughput in multi-hop wireless networks, especially under varying channel conditions. One major problem of relay cooperation is that relaying always incurs energy and possibly delay costs. To a rational and selfish node these costs are worth incurring only if it receives at least comparable returns in the long term. In light of this, we propose a new incentive mechanism called bandwidth exchange $(B E)$ where a node can delegate a portion of its bandwidth to another node in exchange for relay cooperation. In this paper we specifically study $B E$ in the simple form of exchanging orthogonal frequency bands to provide incentives for relaying in a wireless network. Other forms of exchanging bandwidth such as delegation of time-slots or using spreading codes of different lengths are also possible. Using a Nash Bargaining framework, we explore the advantage of $B E$ in both static and fading channels.
\end{abstract}

\section{INTRODUCTION}

Relay cooperation incurs significant costs in energy and delay for the relaying node. In a wireless network of many nodes the greatest immediate benefits may not necessarily go to the nodes that bear the greatest immediate costs. Over time, such costs and benefits may equalize, but there is no reason to assume that nodes will willingly bear a significant and immediate cost for a speculative future benefit. In fact, the rational selfish nature of nodes leading to a network break-down has been observed [1]. Proper incentive mechanisms are therefore required to facilitate cooperation. These incentive mechanisms can be roughly classified as reputation based mechanisms [1]-[5], credit based mechanisms [6]-[9], network assisted pricing [10] [11] and mechanisms based on forwarding games [12]-[15]. These prior efforts often mimic the operation of a complex economy, and in doing so they illustrate the difficulties inherent in this approach. The efficient operation of a complex economy requires such enablers as a stable currency, a system of credit and credit-worthiness, a shared understanding of what things are worth, and a good deal of record keeping. It may be that this complexity is not warranted here, and that a simpler approach, based on the mechanisms of bartering certain wireless resources can provide the necessary incentives for the degree of cooperation we require.

In this paper we propose an autonomous node delegating a portion of its bandwidth to another node in exchange for relay cooperation, which we call bandwidth exchange $(B E)$, as a natural choice for such kind of incentive mechanisms.
$B E$ can be realized in a number of ways such as reassigning time slots, choosing spreading codes of different lengths and even exchanging orthogonal frequency bands. We study in detail $B E$ in the form of exchanging orthogonal frequency bands to provide incentives for relaying in a wireless network. We discuss the $B E$ mechanism using the framework of Nash Bargaining.

\section{SYSTEM MODEL}

We consider $N$ nodes communicating to an access point (AP), each using a nonoverlapping bandwidth $W_{i}$ and transmit power $P_{i}^{T}, i=1,2, \ldots, N$. The channel gain of link $i j$ is denoted by $\rho_{i j}\left(=\rho_{j i}\right)$ and is modeled as

$$
\rho_{i j}=\kappa d_{i j}^{-3}, \quad i=1,2, \ldots, N, j=0,1,2, \ldots, N,
$$

where $d_{i j}$ is the distance between node $i$ and $j, \kappa=6 \times$ $10^{6} \mathrm{MHz} \cdot \mathrm{m}^{3} / \mathrm{mW}$ is the path loss constant and the AP is labeled with index 0 . In a fading environment $\kappa$ is a random variable that makes $\rho_{i j}$ a random variable. For example, under Rayleigh fading $\kappa$ assumes an exponential distribution and so does $\rho_{i j}$

$$
p\left(\rho_{i j}\right)=\frac{1}{\bar{\rho}_{i j}} \exp \left(-\frac{\rho_{i j}}{\bar{\rho}_{i j}}\right)
$$

where $\bar{\rho}_{i j}$ is the mean.

We assume that each node has an infinite amount of data to transmit with a minimum data rate requirement $R_{i}^{\text {min }}$. Initially a source node $i$ attempts to transmit directly to the AP. If the direct link capacity is less than $R_{i}^{\text {min }}$, it seeks a relay node $j$ which could help forward the data to the AP, possibly with $B E$ to be discussed later. In either case, we assume there is no flow splitting and each link is running at its capacity. For example, when node $i$ uses the direct link, the capacity will be

$$
R_{i 0}=W_{i} \log _{2}\left(1+\frac{\rho_{i 0} P_{i}^{T}}{W_{i}}\right)(\text { bits/second }) .
$$

\section{A. Bandwidth Exchange}

The idea of $B E$ can be best explained in the context of a two-node network (say node 1 and 2 ) potentially cooperating with each other to reach the AP. Let us assume that the channels are quasi-static and we can simply consider the snapshots of them. In each snapshot, node $i(=1,2)$ will request relay cooperation from the other node $j$ only if 
$R_{i 0}<R_{i}^{\min }$ and $R_{i j} \geq R_{i}^{\min }$. Further node $i$ will never grant an incoming request if by cooperation its rate to the AP drops below $R_{i}^{\text {min }}$. Regardless of whether cooperation occurs or not, define node $i$ 's payoff $U_{i}$ as the rate from node $i$ to the AP (via direct transmission or relaying) if it is greater than $R_{i}^{\min }$, or 0 otherwise. That is, when cooperation does not occur

$$
U_{i}=U_{i}^{N C}=\left\{\begin{array}{ll}
R_{i 0}, & R_{i 0} \geq R_{i}^{\min }, \\
0, & R_{i 0}<R_{i}^{\min },
\end{array} \quad i=1,2 .\right.
$$

Note this is the payoff if generated relay request is denied or no relay request is generated. If cooperation occurs, the payoffs achieved by the two nodes will be determined by $B E$. Without loss of generality, suppose the request from node 1 is granted by node 2 , then

$$
\begin{aligned}
& U_{1}=U_{1}^{C}=R_{1}^{m i n}=\left(W_{1}-\Delta W_{1}\right) \log \left(1+\frac{\rho_{12} P_{1}^{T}}{W_{1}-\Delta W_{1}}\right), \\
& U_{2}=U_{2}^{C}=\left(W_{2}+\Delta W_{1}\right) \log \left(1+\frac{\rho_{20} P_{2}^{T}}{W_{2}+\Delta W_{1}}\right)-R_{1}^{m i n}
\end{aligned}
$$

where $\Delta W_{1}$ is the portion of bandwidth that is delegated by node 1 to node 2 under $B E$. Similarly, the payoff for node 1 granting the request from node 2 can be calculated in a symmetric way. As we can see, the basic idea of $B E$ is that whenever a node requests relay cooperation it spontaneously lowers the rate to the minimum required level and delegates the maximally possible extra bandwidth to the relay node as an incentive for cooperation. If cooperation occurs, the relay in return guarantees this minimum rate and uses the rest of the link capacity for its own data.

\section{The Two-Node Relay Problem}

In the setting described above, we see that there could be win-win situations when the relay receives extra bandwidth by serving the source node. From (4)-(6), we define $-u_{i}=$ $U_{i}-U_{i}^{N C}$ as the payoff gain for node $i(i=1,2)$. Then, under cooperation the source acquires a payoff gain of $R_{i}^{\min }$ and the relay acquires a payoff gain of $-u_{i}=U_{i}^{C}-U_{i}^{N C}$. Under no cooperation the payoff gains are zero for both of them. Since cooperation does not occur for snapshots where no relay request is generated, we can focus only on the snapshots where a relay request is generated. With this notion we recognize that the payoff gain in each snapshot is also the instantaneous payoff if we regard a snapshot as the stage of a repeated game in which a node generates a request and the other node decides if it wants to cooperate. This idea is depicted in Fig. 1 where for simplicity we assume $R_{1}^{\min }=R_{2}^{\min }=u_{t}$. We use $P$ and $1-P$ to respectively denote the conditional probabilities with which either node 1 or node 2 requests relay cooperation given that there is a request generated in that stage. $P$ is calculated as

$$
P=P_{2} /\left(P_{1}+P_{2}\right)
$$

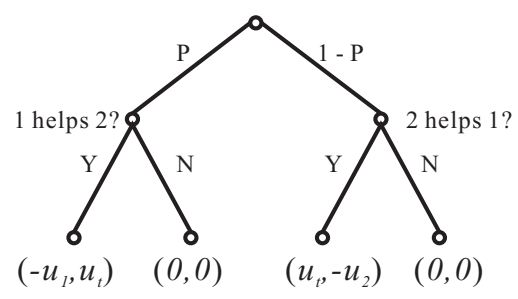

Fig. 1. Extensive form of the stage game of a two-node network.

where

$$
\begin{aligned}
& P_{1}=\iiint_{\substack{R_{10}<R_{1}^{\text {min }} \\
R_{12} \geq R_{1}^{\text {min }} \\
U_{2}^{C} \geq R_{2}^{\text {min }}}} p\left(\rho_{10}, \rho_{20}, \rho_{12}\right) \mathrm{d} \rho_{10} \mathrm{~d} \rho_{20} \mathrm{~d} \rho_{12}, \\
& P_{2}=\iiint_{\substack{R_{20}<R_{2}^{\min } \\
R_{21} \geq R_{2}^{\min } \\
U_{1}^{C} \geq R_{1}^{\text {min }}}} p\left(\rho_{10}, \rho_{20}, \rho_{12}\right) \mathrm{d} \rho_{10} \mathrm{~d} \rho_{20} \mathrm{~d} \rho_{12} .
\end{aligned}
$$

where $p\left(\rho_{10}, \rho_{20}, \rho_{12}\right)$ is the joint PDF of the link gains between node 1 and the AP, node 2 and the AP, node 1 and node 2. Note $P_{i}, i=1,2$ are the unconditional probabilities with which node $i$ requests relay cooperation.

\section{A. The Static Channel Case}

In the case of static (deterministic) channels, $\left(\rho_{10}, \rho_{20}, \rho_{12}\right)$ are modeled as constants across all snapshots. In this case, depending on the path losses of various links, it follows that either $P=0$ or $P=1$. Without loss of generality, assume $P=0$, i.e., node 1 cannot reach the AP unless node 2 provides relay cooperation. Therefore the only viable strategy for node 1 is to request for relay cooperation. For node 2 , it will cooperate if $u_{2}<0$ or will not cooperate if $u_{2}>0$. For any choice of $\left(\rho_{10}, \rho_{20}, \rho_{12}\right)$, node 2 either cooperates or does not cooperate. Let us use $\Omega_{\rho} \subset \mathbb{R}_{+}^{3}$ denote the set of link gains where node 2 cooperates. According to (1), for fixed locations of node 2 and the AP in the 2-dimensional plane, each location $\left(x_{1}, y_{1}\right)$ of node 1 corresponds to a triplet $\left(\rho_{10}, \rho_{20}, \rho_{12}\right)$. This translates $\Omega_{\rho}$ into a geometric region $\mathcal{A}_{12}$ in which upon the request of node 1 , node 2 always provides relay cooperation. To determine $\mathcal{A}_{12}$, note

$$
\mathcal{A}_{12}=\mathcal{A}_{12}^{1} \bigcap \mathcal{A}_{12}^{2} \bigcap \mathcal{A}_{12}^{3},
$$

where

$$
\begin{aligned}
& \mathcal{A}_{12}^{1}=\left\{\left(x_{1}, y_{1}\right): R_{10}<R_{1}^{\text {min }}\right\}, \\
& \mathcal{A}_{12}^{2}=\left\{\left(x_{1}, y_{1}\right): R_{12} \geq R_{1}^{\text {min }}\right\}, \\
& \mathcal{A}_{12}^{3}=\left\{\left(x_{1}, y_{1}\right): U_{2}^{C} \geq U_{2}^{N C}\right\} .
\end{aligned}
$$

Fig. 2 shows a numerical example for the two-node network using $B E$ in a static channel model based on (11a)-(11c). We assume the AP is fixed at the origin in the 2-dimensional plane and node 2 fixed at $(-100 \mathrm{~m}, 0) ; P_{1}^{T}=P_{2}^{T}=100 \mathrm{~mW}, W_{1}=$ $W_{2}=20 \mathrm{MHz}, R_{1}^{\min }=R_{2}^{\min }=30 \mathrm{Mbps}$. In the bright area $\mathcal{A}_{12}$ the request for relay cooperation from node 1 will be granted; in the dark area either no request will be generated or any request will be declined by node 2 . In this sense, $\mathcal{A}_{12}$ 
can be called the cooperation region. Note that in the absence of $B E$, the cooperation region is empty because $\mathcal{A}_{12}^{3}$ in (11c) is empty.

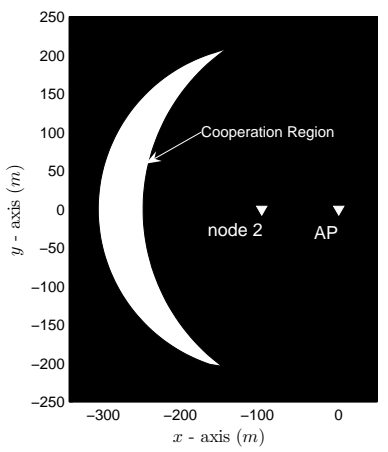

Fig. 2. In a static channel node 1 uses node 2 as relay in the bright area.

\section{B. The Fading Channel Case}

For the fading channel case, the set of link gains $\rho_{i j}$ change from one snapshot to another according to the exponential distribution given by (2). We model the situation as a repeated game between the two nodes. Fig. 1 shows one stage of the game assuming $R_{1}^{\min }=R_{2}^{\min }=u_{t}$. Note that unlike the static channel case, $P$ will take on a value between 0 and 1 and both nodes have a chance to either generate relay requests or provide relay cooperation. The game can be rewritten in its normal form as shown in Table I, from which we immediately have the following observations.

TABLE I

NORMAL FORM OF THE STAGE GAME.

\begin{tabular}{|c|c|c|}
\hline 1 & Cooperation (C) & Noncooperation (N) \\
\hline $\mathrm{C}$ & $\left(-P u_{1}+(1-P) u_{t}, P u_{t}-(1-P) u_{2}\right)$ & $\left(-P u_{1}, P u_{t}\right)$ \\
\hline $\mathrm{N}$ & $\left((1-P) u_{t},-(1-P) u_{2}\right)$ & $(0,0)$ \\
\hline
\end{tabular}

Proposition 1: If $u_{1}<0$ the efficient strategy for node 1 is to cooperate; if $u_{2}<0$ the efficient strategy for node 2 is to cooperate.

Proof: According to Table I, if $u_{1}<0$, cooperation will be the dominant strategy for node 1 . The dominant strategy for node 2 will be cooperation if $u_{2}<0$ or noncooperation if $u_{2} \geq 0$. Likewise, if $u_{2}<0$, then cooperation will be the dominant strategy for 2 . The dominant strategy of 1 will be cooperation if $u_{1}<0$ or noncooperation if $u_{1} \geq 0$. In both cases $\left(u_{1}<0\right.$ or $\left.u_{2}<0\right)$, the outcome payoffs strictly dominates $(0,0)$, thereby being efficient.

Proposition 1 allows us to focus on the case where $u_{1}, u_{2} \geq$ 0 , i.e., both node 1 and node 2 suffer an expected loss by providing relay cooperation. In the absence of $B E$ the potential relay has to apportion a part of its original capacity to sustain the minimum required rate of the source, i.e., the payoff loss $\left(u_{1}\right.$ or $\left.u_{2}\right)$ will be

$$
u_{1}=u_{2}=u_{t}
$$

With $B E$, where the source node delegates bandwidth to the relay, the payoff loss is upper bounded by

$$
0 \leq u_{1}, u_{2} \leq u_{t}
$$

Thus $B E$ provides a mechanism that serves to reduce the payoff loss, thereby providing stronger incentive for cooperation.

In general, we wish to avoid the apparent inefficient Nash equilibrium (where both nodes choose not to cooperate) in the stage game in a number of situations where cooperation is a more desirable outcome. If the stage game is played repeatedly, the Folk Theorem [16] [17] can be invoked to characterize subgame perfect equilibria as long as they strictly dominate $(0,0)$. However, there still remains the issue of which specific equilibrium is most desirable. We will seek an optimal equilibrium in the sense of a Nash Bargaining Solution (NBS), which possesses the advantages of both efficiency and proportional fairness. Using this approach we investigate under what conditions the two nodes will be fully cooperative, cooperative but with some reservation, or completely noncooperative.

The NBS is defined through a set of axioms and is unique. If noncooperation means zero payoff for everyone (as in our discussion of the two-node network), the calculation of a $\mathrm{N}$ player NBS boils down to solving the following optimization problem:

$$
\begin{array}{ll}
\underset{v_{i}, i=1,2, \ldots, N}{\operatorname{maximize}} & \prod_{i=1}^{N} v_{i} \\
\text { subject to } & \left(v_{1}, v_{2}, \ldots, v_{N}\right) \text { feasible, }
\end{array}
$$

where $\left(v_{1}, v_{2} ., \ldots, v_{N}\right)$ is the payoff vector of the $N$ players for a feasible strategy profile, which is realized by mixing the available pure strategy profiles with certain probabilities. For our two-node network, if we define $\left(\tilde{v}_{1}^{j}, \tilde{v}_{2}^{j}\right)$ as the payoff of the $j$ th pure strategy profile, i.e.,

$$
\begin{aligned}
\left(\tilde{v}_{1}^{1}, \tilde{v}_{2}^{1}\right) & =\text { payoffs for }\langle N, N\rangle=(0,0), \\
\left(\tilde{v}_{1}^{2}, \tilde{v}_{2}^{2}\right) & =\text { payoffs for }\langle N, C\rangle=\left((1-P) u_{t},-(1-P) u_{2}\right), \\
\left(\tilde{v}_{1}^{3}, \tilde{v}_{2}^{3}\right) & =\text { payoffs for }\langle C, C\rangle \\
& =\left(-P u_{1}+(1-P) u_{t}, P u_{t}-(1-P) u_{2}\right), \\
\left(\tilde{v}_{1}^{4}, \tilde{v}_{2}^{4}\right) & =\text { payoffs for }\langle C, N\rangle=\left(-P u_{1}, P u_{t}\right),
\end{aligned}
$$

then we can explicitly write out the corresponding optimization problem for the two-node network as

$$
\begin{array}{cl}
\underset{\lambda_{i}, i=1,2,3,4}{\operatorname{maximize}} & v_{1} v_{2} \\
\text { subject to } & v_{1}=\lambda_{1} \tilde{v}_{1}^{1}+\lambda_{2} \tilde{v}_{1}^{2}+\lambda_{3} \tilde{v}_{1}^{3}+\lambda_{4} \tilde{v}_{1}^{4}, \\
& v_{2}=\lambda_{1} \tilde{v}_{2}^{1}+\lambda_{2} \tilde{v}_{2}^{2}+\lambda_{3} \tilde{v}_{2}^{3}+\lambda_{4} \tilde{v}_{2}^{4}, \\
& \lambda_{i} \geq 0, \quad i=1,2,3,4, \\
& \lambda_{1}+\lambda_{2}+\lambda_{3}+\lambda_{4}=1
\end{array}
$$

where $\lambda_{i}, i=1,2,3,4$ are the mixing probabilities. However, it turns out we can either solve (15) or take a much simpler geometric approach to derive the NBS for the two-node network under various channel conditions. For the latter approach, 
denote the four points $\left(\tilde{v}_{1}^{j}, \tilde{v}_{2}^{j}\right), j=1,2,3,4$ by $O, A, B, C$ on the $v_{1} v_{2}$ plane with $O$ coinciding with the origin. The NBS is characterized by the following facts [18]:

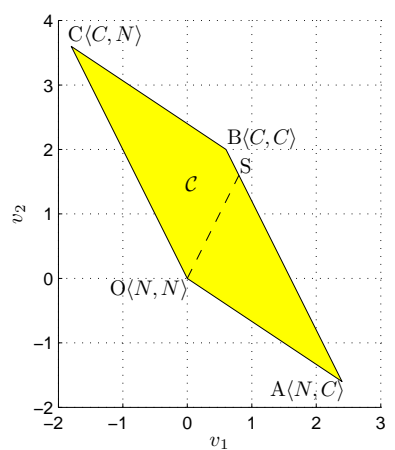

Fig. 3. A geometric interpretation of the NBS (denoted by $S$ ) which is on the Pareto boundary $\partial \mathcal{C}$ such that the slope of $O S$ is the negative of the slope of $A B$.

1) The feasible set $\mathcal{C}$ of $\left(v_{1}, v_{2}\right)$ is convex.

2) There is a unique NBS, denoted by $S$, sitting on the boundary $\partial \mathcal{C}$ of the feasible set in the first quadrant.

3 ) If we denote the origin as $O$, then the negative slope of segment $O S$ is equal to the gradient of $\partial \mathcal{C}$ at $S$.

These facts enable us to determine the NBS geometrically, as exemplified by Fig. 3 .

Proposition 2: If $u_{1}, u_{2}>0$, the feasible set $\mathcal{C}$ is contained within a parallelogram in the $v_{1} v_{2}$ plane with vertices being the points $O, A, B, C$. Further, $S$ is always on the segment $A B$ or $B C$ and can be determined by the following conditions

$$
\begin{aligned}
\tan B<-\frac{u_{t}}{u_{1}} & \text { or } \tan B>\frac{u_{t}}{u_{1}} \Longleftrightarrow S \text { on } A B, \\
-\frac{u_{2}}{u_{t}}<\tan B<\frac{u_{2}}{u_{t}} \Longleftrightarrow S \text { on } B C, & \Longleftrightarrow S \text { at } B .
\end{aligned}
$$

where $\tan B$ denotes the slope of the segment $O B$.

Proposition 2 provides an exact procedure to determine the location of $S$. Since the parallelogram $O A B C$ is the convex hull of payoffs of four pure strategy profiles, any point in the parallelogram is to be realized with a set of mixing probabilities. When $S$ is on $A B$ it means when node 1 requests relay cooperation from node 2 , it is always granted but when node 2 requests relay cooperation from node 1 it is granted with a probability $P_{C}$. We can determine $P_{C}$ by solving the following equations

$$
\begin{aligned}
S & =P_{C} \cdot B+\left(1-P_{C}\right) \cdot A, \\
\tan S & =-\tan \angle A B=\frac{u_{t}}{u_{1}} .
\end{aligned}
$$

where $\tan \angle A B$ denotes the slope of the segment $A B$. The conclusion when $S$ lies on $B C$ is similar. Only when $S$ coincides with $B$ will both nodes unconditionally grant the relay cooperation requests for each other.
Before proceeding to various special cases of the two-node network with $B E$, we first state the following result which follows from Proposition 2. Specifically, we consider the NBS when there is no $B E$, i.e., $\Delta W_{i}=0, i=1,2$.

Proposition 3: When there is no $B E$ and $R_{1}^{\text {min }}=R_{2}^{\text {min }}=$ $u_{t}$, the optimal strategies for the two nodes will be to not cooperate.

We now consider several special cases of the NBS when $B E$ is used as an incentive for relaying in a two-node network.

1) $P=0.5, u_{t} \geq u_{1}=u_{2} \geq 0$ : This case corresponds to a scenario where the channel conditions are such that each node is equally likely to ask the other node for relay cooperation; further, the loss in payoff due to cooperation for each node is identical in every snapshot. Using (16) we know $S=B$ in this case. In fact, this is where two nodes have symmetric bargaining power and we expect them to adopt the same optimal strategy. Since $\langle C, C\rangle$ dominates $\langle N, N\rangle$, they always cooperate. Simple as it is, this case reflects many practical wireless networking scenarios where many nodes with similar radio capabilities experience statistically similar fading conditions. The results show that cooperation is always an optimal strategy for every node in the sense of NBS.

2) $P=0.5, u_{t} \geq u_{1}>u_{2} \geq 0$ : This case corresponds to a scenario where the channel conditions are such that each node is equally likely to ask the other node for relay cooperation; however, node 1 has a greater payoff loss when it grants a request for relay cooperation from node 2 in every snapshot. Thus an apparent asymmetry is introduced into the bargaining powers of the nodes in terms of expected loss. We look into the case $u_{1}>u_{2}$; the case $u_{1}<u_{2}$ is similar. For this special case we have the following conclusion.

Proposition 4: When $P=0.5$ and $u_{t} \geq u_{1}>u_{2} \geq 0$, node 2 always cooperates; node 1 cooperates if

$$
u_{1} \leq \frac{u_{t}^{2}}{2 u_{t}-u_{2}}
$$

is satisfied. Otherwise node 1 will cooperate with probability $P_{C}$ and not cooperate with probability $1-P_{C}$ where

$$
P_{C}=\frac{1}{2}\left(\frac{u_{t}}{u_{1}}+\frac{u_{2}}{u_{t}}\right) .
$$

Proof: When $P=0.5, u_{t} \geq u_{1}>u_{2} \geq 0$,

$$
\tan B=\frac{u_{t}-u_{2}}{u_{t}-u_{1}}=\frac{1-u_{2} / u_{t}}{1-u_{1} / u_{t}}=\frac{1-\lambda_{2}}{1-\lambda_{1}},
$$

where we define

$$
0 \leq \lambda_{2}=\frac{u_{2}}{u_{t}}<\lambda_{1}=\frac{u_{1}}{u_{t}} \leq 1 .
$$

It is simple to show $1 /\left(2-\lambda_{1}\right) \geq \lambda_{1}>\lambda_{2}$, which implies

$$
\tan B=\frac{1-\lambda_{2}}{1-\lambda_{1}} \geq \lambda_{2}=\frac{u_{2}}{u_{t}} .
$$

Therefore, according to (16), $S$ will never lie on the segment $B C$ and node 2 should always take cooperation as the only pure strategy, though node 1 in certain situations could mix cooperation and noncooperation strategies. To determine in 
what situations node 1 will also take cooperation as the only pure strategy we need to find the conditions under which $S$ coincides with $B$. This amounts to solving the inequality $\tan B \leq u_{t} / u_{1}=1 / \lambda_{1}$. The solution is

$$
\lambda_{1} \leq \frac{1}{2-\lambda_{2}} \text { or } u_{1} \leq \frac{u_{t}^{2}}{2 u_{t}-u_{2}} .
$$

When (23) is not satisfied, node 1 will mix cooperation and noncooperation with probability $P_{C}$ and $1-P_{C}$ respectively. $P_{C}$ given in (19) is obtained by solving (17).

To summarize, Proposition 4 suggests that when the expected losses are almost the same, both nodes will fully cooperate; but when there is a significant difference, the node with greater expected loss will mix cooperation and noncooperation strategies.

3) $P>0.5, u_{t} \geq u_{1}=u_{2} \geq 0$ : This case corresponds to the scenario where the channel conditions are such that node 2 requests relay cooperation more often than node 1; however, the loss in payoff due to cooperation is similar in every snapshot. This is another way to introduce asymmetry into their bargaining powers of the two nodes. For example, we study the case $P>0.5$ and $u_{1}=u_{2}=u$ with the following results.

Proposition 5: If $u_{t} \geq u_{1}=u_{2}=u \geq 0$ and $P>0.5$, node 2 will always cooperate; node 1 will cooperate if

$$
P \leq \frac{u^{2}+u_{t}^{2}}{\left(u+u_{t}\right)^{2}}
$$

is satisfied. When it is not satisfied, node 1 will cooperate with probability $P_{C}$ and not cooperate with $1-P_{C}$ where $P_{C}$ is given by

$$
P_{C}=\frac{1-P}{2 P}\left(\frac{u_{t}}{u}+\frac{u}{u_{t}}\right) .
$$

Proof: If $-P u+(1-P) u_{t}<0$, we can verify

$$
\tan B<-\frac{u_{t}}{u} .
$$

Referring to (16), this implies $S$ lies in the interior of segment $A B$. If $-P u+(1-P) u_{t}>0$, since $P>0.5$, we have

$$
P u_{t}^{2}+P u^{2}>2(1-P) u_{t} u,
$$

which implies

$$
\frac{P u_{t}-(1-P) u}{-P u+(1-P) u_{t}}>\frac{u}{u_{t}} .
$$

Then (16) implies $S$ coincides with $B$ or is in between $A$ and $B$. Therefore, when $P>0.5$, node 2 should always take cooperation as the only pure strategy, though node 1 in certain situations could mix cooperation and noncooperation strategies. To determine in what situations node 1 will also take cooperation as the only pure strategy we need to find the conditions under which $S$ coincides with $B$. This amounts to solving the inequalities

$$
\frac{P u_{t}-(1-P) u}{-P u+(1-P) u_{t}} \leq \frac{u_{t}}{u} \text { and }-P u+(1-P) u_{t}>0 .
$$

The solution is then given by (24). $P_{C}$ obtained by solving (17) is shown as in (25).

To summarize Proposition 5 suggests when the two nodes request for relay cooperation at almost the same rate, both nodes will fully cooperate; but when there is a significant difference, the node who sends less requests will mix cooperation and noncooperation strategies.

\section{NUMERICAl RESUltS FOR FADING CHANNELS}

In this section we present numerical results for a twonode network cooperating via $B E$ in a fading channel. We assume that the fading channel gains are given according to the model in (1) and (2). Throughout this section, we consider a rectangular area that is $400 \times 600 \mathrm{~m}^{2}$. We assume that the AP is fixed at the origin in the 2-dimensional plane and node 2 fixed at $(-100 \mathrm{~m}, 0)$. The relevant communication parameters are chosen to be $P_{1}^{T}=P_{2}^{T}=100 \mathrm{~mW}, W_{1}=W_{2}=20 \mathrm{MHz}$, $R_{1}^{\text {min }}=R_{2}^{\text {min }}=30 \mathrm{Mbps}$. In the following we present results for the NBS strategies under $B E$ for different locations of node 1 in the rectangular area. For simplicity, we partition the area into $25 \times 25 \mathrm{~m}^{2}$ grids with node 1 at the center of each grid.

Fig. 4(a) shows the probability of node 1 requesting relay cooperation from node 2 for different locations of node 1 in the rectangular area. In Fig. 4(b) we show the probability with which node 2 grants the relay cooperation request. It is evident that when node 1 gets away from the AP, it sends more relay cooperation requests to node 2 and for a wide range of locations of node 1 , those requests will be granted with probability 1 . This result illustrates that $B E$ effectively stimulates cooperation.

To appreciate how helpful $B E$ is in improving the performance in fading channels, it is also illuminating to make a comparison with the NBS under the same assumptions except without $B E$. In Fig. 4(c) the coverage area (the possible locations of node 1) where node 1 is guaranteed an outage probability less than $10^{-1}$ is shown in bright color when there is no $B E$. In Fig. 4(d) the corresponding coverage area is shown with a $B E$ based incentive mechanism. Similar results are shown in Fig. 4(e) and 4(f) for an outage probability guarantee of $10^{-2}$. In all cases, it is observed that $B E$ does indeed serve as an effective incentive mechanism by increasing the coverage area of node 1 .

\section{CONCLUSION}

In this paper we studied an incentive mechanism called bandwidth exchange for relay cooperation in a multi-hop ad hoc network to enhance connectivity and throughput. Because relaying possibly incurs energy and delay costs, relay cooperation usually is not guaranteed. However, by delegating a portion of its bandwidth to the potential relay, a source node has a better chance to stimulate relay cooperation. We have shown that for both static and dynamic channels, $B E$ as a simple incentive mechanism triggers wider cooperation. Under the framework of Nash Bargaining, we illustrated the 


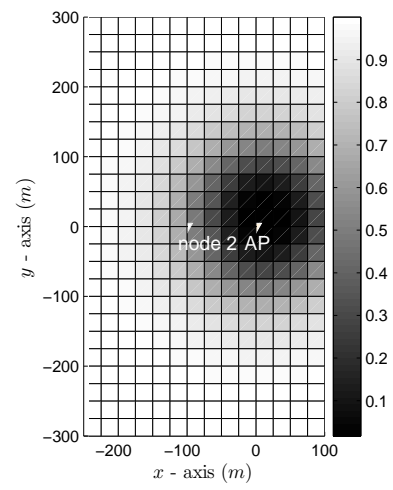

(a)

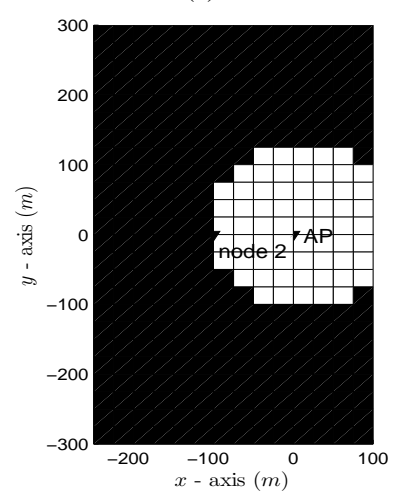

(c)

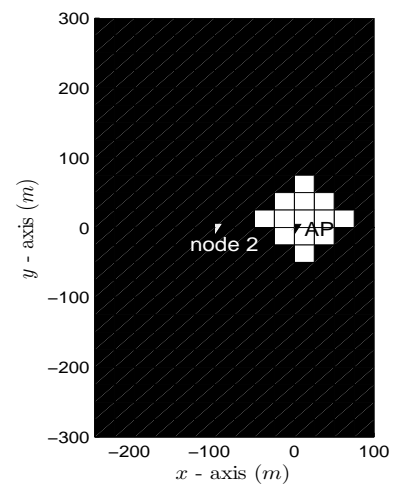

(e)

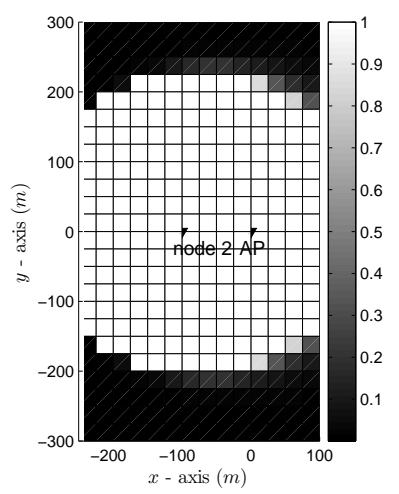

(b)

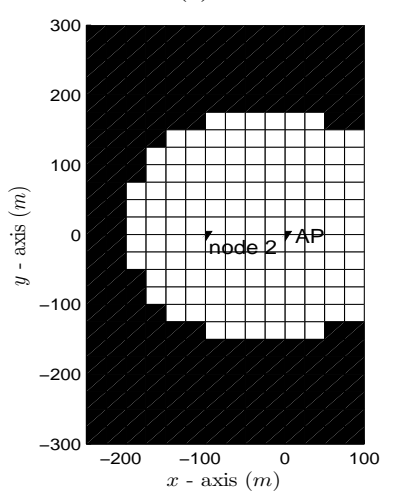

(d)

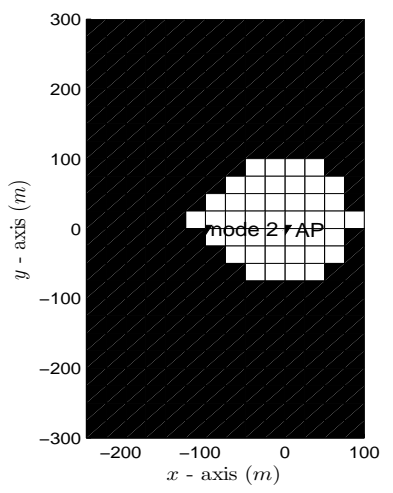

(f)
Fig. 4. The $x y$ plane shows all possible locations of node 1 with node 2 and $\mathrm{AP}$ at fixed locations. (a) probability that node 1 requests relay cooperation from node 2; (b) probability that node 2 grants a relay request; (c) coverage area of node 1 without $B E$ for an outage probability less than $10^{-1}$; (d) coverage area of node 1 with $B E$ for an outage probability less than $10^{-1}$; (e) coverage area of node 1 without $B E$ for an outage probability less than $10^{-2}$; (f) coverage area of node 1 with $B E$ for an outage probability less than $10^{-2}$.

significant improvement it has on the two-node network.

There is a need for studying the behavior of $B E$ in a general $N$-node network where more practical models with traffic will be included. A more sophisticated strategy space to each node, rather than simply cooperation or noncooperation, will also be studied. For example, a source can choose a rate to be supported by the relay and a relay can choose the rate that it guarantees the source. It is of equal interest to extend the concept of $B E$ into relay cooperation of more than two hops and results on some of these issues will be presented in an upcoming journal version of the results presented here.

\section{ACKNOWLEDGMENT}

This work is supported in part by the NSF under grant number CCF-0634973 and CNS-0721826.

\section{REFERENCES}

[1] S. Marti, T. Giuli, K. Lai, and M. Baker, "Mitigating routing misbehavior in mobile ad hoc networks," in Proceedings of The Sixth International Conference on Mobile Computing and Networking, August 2000.

[2] S. Buchegger and J.-Y. Le Boudec, "Self-policing mobile ad hoc networks by reputation systems," IEEE Communications Magazine, pp. 101-107, July 2005.

[3] Q. He, D. Wu, and P. Khosla, "Sori: A secure and objective reputationbased incentive scheme for ad hoc networks," in Proceedings of IEEE Wireless Communications and Networks Conference (WCNC), March 2004.

[4] Y. Liu and Y. R. Yang, "Reputation propagation and agreement in mobile ad-hoc networks," in Proceedings of IEEE Wireless Communications and Networks Conference (WCNC), March 2003.

[5] P. Michiardi and R. Molva, "Core: A cooperative reputation mechanism to enforce node cooperation in mobile ad hoc networks," in Communications and Multimedia Security Conference (CMS), 2002.

[6] L. Buttyan and J. P. Hubaux, "Enforcing service availability in mobile ad-hoc wans," in Proceedings of IEEE/ACM Workshop on Mobile Ad Hoc Networking and Computing (MobiHOC), August 2000.

[7] — "Nuglets: A virtual currency to stimulate cooperation in selforganized mobile ad-hoc networks," Swiss Federal Institute of Technology, Technical report, January 2001.

[8] — , "Stimulating cooperation in self-organizing mobile ad hoc networks," ACM Journal for Mobile Networks (MONET), October 2003, special issue on Mobile Ad Hoc Networks.

[9] S. Zhong, J. Chen, and Y. R. Yang, "Sprite: A simple, cheat proof, credit-based system for mobile ad-hoc networks," in Proceedings of IEEE INFOCOM'03, April 2003.

[10] O. Ileri, S.-C. Mau, and N. Mandayam, "Pricing for enabling forwarding in self-configuring ad hoc networks," IEEE Journal on Selected Areas in Communications, vol. 23, pp. 151-162, January 2005, special Issue on Wireless Ad Hoc Networks.

[11] M. Lindstrom and P. Lungaro, "Resource delegation and rewards to stimulate forwarding in multihop cellular networks," in Proceedings of IEEE Vehicular Technology Conference (VTC), vol. 4, June 2005, pp. 2152-2156.

[12] L. Anderegg and S. Eidenbenz, "Ad hoc vcg: A truthful and cost-efficient routing protocol for mobile ad hoc networks with selfish agents," in Proceedings of IEEE Mobicom, April 2003.

[13] M. Felegyhazi, J. P. Hubaux, and L. Buttyan, "Nash equilibria of packet forwarding strategies in wireless ad hoc networks," IEEE Transactions on Mobile Computing, vol. 5, pp. 463-475, May 2006.

[14] P. Michiardi and R. Molva, "Game theoretic analysis of security in mobile ad hoc networks," Institut Eurecom, Technical report, April 2002.

[15] S. Zhong, L. E. Li, Y. Liu, and Y. R. Yang, "On designing incentivecompatible routing and forwarding protocols in wireless ad-hoc networks - an integrated approach using game theoretical and cryptographic techniques," in Proceedings of IEEE Mobicom, April 2003.

[16] J. Friedman, "A noncooperative equilibrium for supergames," Rev. Econ. Stud., vol. 38, pp. 1-12, 1971.

[17] D. Fudenberg and E. Maskin, "The folk theorem in repeated games with discounting or with incomplete information," Econometrica, vol. 54, no. 3, pp. 533-554, May 1986.

[18] G. Owen, Game Theory, 3rd ed. Academic Press, 1995. 